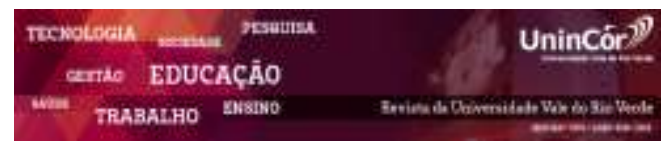

Revista da Universidade Vale do Rio Verde ISSN: 1517-0276 / EISSN: 2236-5362

Vol. 16 | n. 1 | Ano 2018

Yasmin Ferreira Alves

Universidade Paulista - UNIP

yasminalvex@hotmail.com

Caroline Regina da Silva Universidade Paulista - UNIP carolineregina1@gmail.com

Mônica Glória Neumann Spinelli Universidade Paulista - UNIP monicaspi404@gmail.com

\section{TEOR DE SÓDIO E CONTRIBUIÇÃO CALÓRICA DE ALIMENTOS \\ ULTRAPROCESSADOS NO CARDÁPIO \\ DE UMA UNIDADE DE ALIMENTAÇÃ̃O \\ E NUTRIÇÃO ESCOLAR EM SÃO \\ PAULO - SP}

\title{
RESUMO
}

Além da alimentação domiciliar, a criança e o adolescente consomem a alimentação escolar fornecida pela instituição na qual estudam. Diante disso, o objetivo desse estudo foi analisar a quantidade de alimentos ultraprocessados adquiridos em uma UAN escolar na cidade de São Paulo e avaliar a densidade energética e o teor de sódio desses produtos ofertados no lanche da tarde para com o total calórico da refeição. Foram coletados dados de rastreabilidade de dois meses do estoque da unidade, com o objetivo de obter a relação de produtos utilizados neste período. Os alimentos foram classificados em quatro grupos: in natura e minimamente processados, processados, ultraprocessados e ingredientes culinários. Posteriormente, foi realizado o cálculo do valor calórico total referente ao lanche da tarde, de duas semanas não consecutivas e analisada a contribuição da densidade calórica e quantidade de sódio dos produtos ultraprocessados ofertados nessa refeição. Nos dois meses avaliados, foram contabilizados 128 e 125 itens consecutivamente. Em média 46,6\% dos itens foram classificados como ultraprocessados. Com relação à contribuição energética, a porcentagem de quilocalorias provenientes de ultraprocessados em relação ao valor calórico total do lanche da tarde foi de aproximadamente $57 \%$, enquanto que a média de porcentagem de sódio proveniente de ultraprocessados foi de aproximadamente $74 \%$. Cabe aos serviços de alimentação a adequação do cardápio, para que haja a oferta de preparações equilibradas, com o objetivo de proteger à saúde dos comensais, estimular hábitos alimentares mais saudáveis, principalmente na infância, pois sabe-se que a escola possui grande influência na formação dos hábitos alimentares das crianças.

Palavras-chave: Serviços de Alimentação. Alimentos Industrializados. Sódio. Guia Alimentar. Alimentação Escolar.

\section{SODIUM AND CALORIC CONTRIBUTION OF \\ ULTRAPROCESSED FOOD ON THE MENU OF A SCHOOL FOOD SERVICE IN SÃO PAULO-SP}

\author{
ABSTRACT \\ Analyze the amount of ultraprocessedfood purchased in a \\ school service in the city of São Paulo and evaluate the energy \\ density and sodium content of the products offered in the
}


afternoon snack for the total calories meal. Data were collected from two months unity stock traceability, with the aim of obtaining the list of products used in this period. The foods were classified into four groups: natural and minimally processed, processed, ultraprocessed and culinary ingredients. Subsequently, the total caloric value calculation for the afternoon snack, two non-consecutive weeks, the contribution of caloric density was examined and amount of sodium ultraprocessedproducts offered in this meal. Within two months, were accounted from 125 to 128 items consecutively. On average $46.6 \%$ of items were classified as ultraprocessed. With regards of energy, the percentage contribution of kilocalories from ultraprocessed in relation to the total calorie afternoon snack was approximately $57 \%$, while the average percentage of sodium from ultraprocessedwas approximately $74 \%$. The food service fited the menu, so the offer of balanced preparations, with the goal of protecting the health of Diners, encourage healthier eating habits, especially in childhood, because it is known that the school has great influence in the formation of the eating habits of children.

Keywords: Food Services. Industrialized Foods. Sodium. Food Guide. School Feeding.

Recebido em: 13/12/2016 - Aprovado em: 05/02/2018 - Disponibilizado em: 15/07/2018

\section{INTRODUÇÃO}

A alimentação cumpre um papel fundamental durante todo o ciclo de vida dos indivíduos. Entre as diferentes fases da vida, pode-se destacar a criança em idade escolar e a adolescência, pois nesse período, os hábitos alimentares adquiridos podem perpetuar até a fase adulta (PAIVA, 2009).

Além da alimentação domiciliar, a criança e o adolescente consomem a alimentação escolar fornecida pela instituição na qual estudam. Assim, as escolas podem ofertar uma ou mais refeições, dependendo do período em que o aluno permanece na escola, que podem ser advindas do Programa Nacional de Alimentação Escolar (PNAE) nas escolas públicas, por autogestão ou por empresa terceirizada nas instituições privadas. Independente disso, a alimentação deve ser equilibrada como qualquer outra refeição do dia, composta por alimentos nutritivos e de boa aceitação, visando contribuir para o sustento e promoção da saúde dos comensais (FLÁVIO, 2006).

Os estabelecimentos que lidam com produção e distribuição de alimentos para coletividades em escolas atualmente são chamados de UANs escolares (Unidade de Alimentação e Nutrição), e têm como objetivo fornecer refeições balanceadas dentro dos padrões dietéticos e higiênico-sanitários, atendendo às necessidades nutricionais de seus clientes, ao passo que se ajuste dentro dos limites financeiros da instituição (ABREU, SPINELLI, SOUZA PINTO, 2016).

Sabe-se que o Brasil tem apresentado profundas modificações no perfil nutricional de sua população devido à transição nutricional. 
Estudos demonstram que a prevalência de sobrepeso mais que triplicou em crianças de 5 a 9 anos de idade (9,7\% em 1975 e 33,4\% em 2009) e quase quadruplicou para crianças e adolescentes de 10 a 19 anos (5,6\% em 1975 e 20,5\% em 2009) (BRASIL, 2009).

Essas alterações estão intimamente relacionadas às mudanças nos hábitos alimentares, seguindo uma tendência global de aumento do consumo de alimentos com alta densidade energética, ricos em sódio, gorduras e açúcares (VIEROS e MARTINELLI, 2012). A Estratégia Global para Alimentação, Atividade Física e Saúde, da Organização Mundial da Saúde (OMS), enfatiza a necessidade de redução desses alimentos, designados como ultraprocessados segundo a classificação de Monteiro et al. (2014), principalmente no âmbito escolar (BIELEMANN et al. 2015).

Neste contexto, uma UAN escolar deve conciliar a preferência e satisfação do cliente (escola) com a oferta de alimentos e refeições nutricionalmente equilibrados aos escolares (VIEROS e MARTINELLI, 2012). Além disso, as refeições elaboradas nas UANs podem ser utilizadas como ferramentas de educação nutricional, modificando positivamente os hábitos alimentares de seu público-alvo (BUENO et al, 2015).

Diante disso, o objetivo desse estudo foi analisar a quantidade de alimentos ultraprocessados adquiridos em uma UAN escolar na cidade de São Paulo e avaliar a contribuição da densidade energética e do teor de sódio desses produtos ofertados no lanche da tarde para com o total calórico da refeição.

\section{METODOLOGIA}

Trata-se de um estudo observacional transversal, realizado em uma UAN escolar de uma instituição particular localizada na região oeste da cidade de São Paulo que atende 655 alunos matriculados, na faixa etária de 7 a 18 anos. A instituição é caracterizada por atender ao Ciclo I e II do Ensino Fundamental $\left(2^{\circ}\right.$ a $9^{\circ}$ série $)$, bem como o Ensino Médio ( $1^{\circ}$ ao $3^{\circ}$ ano), em período integral, de segunda à sexta-feira, das $8 \mathrm{~h}$ às $17 \mathrm{~h}$.

São ofertadas três refeições diárias (lanche da manhã, almoço e lanche da tarde), com horários pré-estabelecidos para cada faixa etária e turma, preparadas por uma empresa terceirizada, contratada pela instituição.

O tipo de contrato da UAN escolar em questão é mandato. Neste tipo de contrato, o cliente (escola) direciona o padrão da refeição, enquanto a contratada (terceirizada) fornece mão de obra e realiza as compras em nome do cliente. O pagamento para a contratada é feito pelo repasse da totalidade dos valores do serviço, além da adição de um valor correspondente à administração (ALVES, 2005).

A coleta de dados foi realizada durante dois meses em 2016, conforme rastreabilidade do estoque. Os itens foram dispostos em planilhas e agrupados segundo proposta do novo Guia Alimentar da População Brasileira (2014), subdividindo-os em quatro grupos: alimentos in natura e minimamente processados, alimentos processados, alimentos ultraprocessados, além de ingredientes culinários (óleos, gorduras, sal e açúcar). 
Segundo a classificação de Monteiro et al. (2014):

Alimentos in natura: são aqueles obtidos diretamente de plantas ou animais e não sofrem qualquer alteração após deixarem a natureza;

Alimentos minimamente processados: são alimentos in natura que foram submetidos a um processamento mínimo de limpeza, remoção de partes não comestíveis ou indesejáveis entre outros processos similares que não envolvam agregação de sal, açúcar, óleos, gorduras ou outras substâncias ao alimento original, a fim de tornalos mais palatáveis;

Alimentos processados: são aqueles que a indústria adiciona sal, açúcar ou outra substância de uso culinário a alimentos in natura para tornalos duráveis e mais agradáveis ao paladar;

\section{Alimentos ultraprocessados: são} produtos fabricados pela indústria, prontos para consumo, feitos inteiramente ou majoritariamente de substâncias extraídas de alimentos (óleos, gorduras, açúcar, amido, proteínas), derivados de constituintes de alimentos (gorduras hidrogenadas, amido modificado) ou sintetizadas em laboratório com base em matérias orgânicas como petróleo e carvão (corantes, aromatizantes, realçadores de sabor e vários tipos de aditivos) com o objetivo de tornar o alimento atraente, acessível, palatável, com longa vida de prateleira e praticidade; e,

Ingredientes culinários: refere-se ao grupo de óleos, sal, gorduras e açúcar. Estas correspondem às substâncias extraídas e purificadas pela indústria a partir de alimentos in natura ou obtidos direto da natureza, e posteriormente passam por meio de vários processos que alteram radicalmente a natureza do alimento original.

Após classificação dos alimentos segundo esses grupos descritos, foi realizado o cálculo do cardápio referente ao lanche da tarde, de duas semanas não consecutivas, e analisada a contribuição da densidade calórica e quantidade de sódio dos produtos ultraprocessados ofertados nessas refeições.

Os dados foram tabulados em Microsoft Excel 2010 e apresentados em tabelas e gráfico de colunas e confrontados com literatura sobre o assunto. A pesquisa seguiu os procedimentos éticos da Resolução $N^{\circ}$ 510, de 07 de abril de 2016.

\section{RESULTADOS}

No mês de setembro, foram avaliados 128 itens alimentícios, dos quais a maioria foi de produtos in natura e minimamente processados $(46,9 \%)$, seguido de produtos ultraprocessados (43\%). No mês de outubro, foram avaliados 125 itens e nota-se semelhança da quantidade de produtos nos dois meses, bem como baixa quantidade de alimentos processados, como apresentado na Figura I.

Vale salientar que o baixo percentual de ingredientes culinários, deve-se por conta da sua classificação: óleos, sal, açúcar e gorduras. 
Figura I - Percentual de tipos de alimentos segundo Guia Alimentar da População Brasileira (2014) adquiridos no estoque de dois meses de uma UAN escolar. São Paulo, 2016.

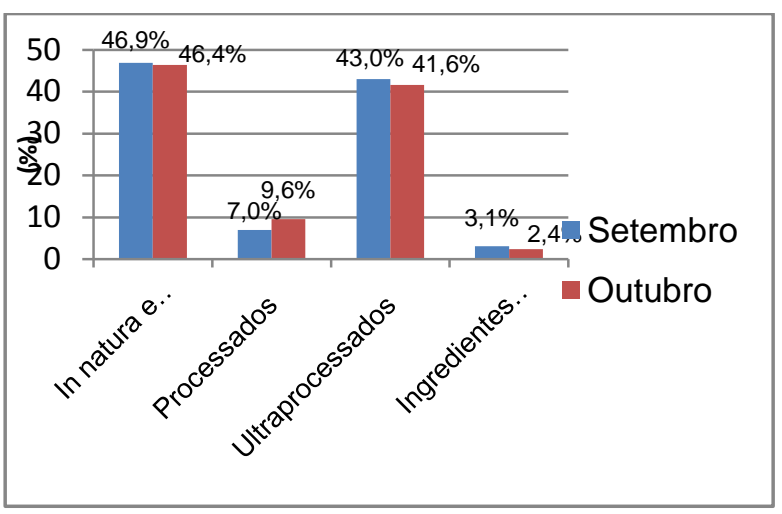

Em relação à contribuição da densidade calórica e quantidade de sódio dos produtos ultraprocessados para com o total da refeição, foram calculados os cardápios do lanche da tarde de duas semanas (Tabela I e II).

Tabela I - Contribuição das calorias e sódio dos produtos ultraprocessados no lanche da tarde de uma semana do mês de setembro, São Paulo,2016.

\begin{tabular}{|c|c|c|c|c|c|}
\hline & Segunda & Terça & Quarta & Quinta & Sexta \\
\hline \multirow[t]{4}{*}{ Alimentos } & $\begin{array}{c}\text { Bolo de } \\
\text { cenoura } \\
\text { com } \\
\text { cobertura } \\
\text { de } \\
\text { chocolate }\end{array}$ & $\begin{array}{l}\text { Lanche } \\
\text { natural* }\end{array}$ & $\begin{array}{l}\text { Torta de } \\
\text { frango }\end{array}$ & $\begin{array}{l}\text { Torrada com } \\
\text { requeijão }\end{array}$ & Biscoito Cookie \\
\hline & $\begin{array}{l}\text { Biscoito } \\
\text { polvilho }\end{array}$ & Melancia & Sequilhos & $\begin{array}{c}\text { Biscoito } \\
\text { amanteigado }\end{array}$ & $\begin{array}{l}\text { Biscoito Club } \\
\text { social }\end{array}$ \\
\hline & Laranja & $\begin{array}{l}\text { Suco de } \\
\text { maracujá }\end{array}$ & $\begin{array}{c}\text { Banana } \\
\text { nanica }\end{array}$ & $\begin{array}{l}\text { Leite com } \\
\text { Ovomaltine }\end{array}$ & Melão \\
\hline & $\begin{array}{l}\text { Suco de } \\
\text { caju }\end{array}$ & & $\begin{array}{l}\text { Suco de } \\
\text { morango }\end{array}$ & Mamão & Suco de abacaxi \\
\hline Total Kcal & 415,8 & 215,9 & 334,7 & 474,3 & 288,4 \\
\hline Total Na (mg) & 57,4 & 249,9 & 234,1 & 517,0 & 296,4 \\
\hline $\begin{array}{l}\text { Total kcal dos } \\
\text { ultraprocessados }\end{array}$ & 65,2 & 152,7 & 136,0 & 368,4 & 261,0 \\
\hline $\begin{array}{l}\text { Total Na (mg) dos } \\
\text { ultraprocessados }\end{array}$ & 20,0 & 199,6 & 62,0 & 397,0 & 294,0 \\
\hline
\end{tabular}

Fonte: Tabela de Composição de Alimentos: TACO, Philippi, Rótulo dos alimentos.

*Composição: pão de forma integral, peito de peru, queijo muçarela, alface crespa. 
Tabela II.Contribuição das calorias e sódio dos produtos ultraprocessados no lanche da tarde de uma semana do mês de outubro. São Paulo, 2016.

\begin{tabular}{|c|c|c|c|c|c|}
\hline & Segunda & Terça & Quarta & Quinta & Sexta \\
\hline \multirow{4}{*}{ Alimentos } & Esfiha de carne & Roll cake & $\begin{array}{c}\text { Pão bisnaga } \\
\text { com presunto e } \\
\text { muçarela }\end{array}$ & Lanche natural $*$ & $\begin{array}{c}\text { Bolo de } \\
\text { chocolate com } \\
\text { cobertura }\end{array}$ \\
\hline & Melancia & Club social & Mamão & $\begin{array}{c}\text { Leite com } \\
\text { achocolatado }\end{array}$ & $\begin{array}{l}\text { Wafer de } \\
\text { morango }\end{array}$ \\
\hline & Suco de laranja & $\begin{array}{l}\text { Vitamina de } \\
\text { morango }\end{array}$ & $\begin{array}{l}\text { Suco de } \\
\text { abacaxi }\end{array}$ & Melão & Abacaxi Havaí \\
\hline & & Laranja & & $\begin{array}{l}\text { Suco de } \\
\text { maracujá }\end{array}$ & Suco de goiaba \\
\hline Total Kcal & 338,0 & 400,9 & 182,2 & 339,1 & 504,6 \\
\hline Total Na (mg) & 730,0 & 380,0 & 302,7 & 309,9 & 123,9 \\
\hline $\begin{array}{l}\text { Total Kcal dos } \\
\text { ultraprocessados }\end{array}$ & 269,0 & 274,0 & 50,6 & 192,8 & 216,2 \\
\hline $\begin{array}{l}\text { Total Na }(\mathrm{mg}) \text { dos } \\
\text { ultraprocessados }\end{array}$ & 730,0 & 250,0 & 155,8 & 140,2 & 71,0 \\
\hline
\end{tabular}

Fonte: Tabela de Composição de Alimentos: TACO, Philippi, Rótulo dos alimentos.

*Composição: pão de forma integral, peito de peru, queijo muçarela, alface crespa.

Verificou-se que, quando analisada a semana como um todo, mais da metade das calorias totais dos cardápios dos lanches são advindas de alimentos ultraprocessados, e mais de $70 \%$ do sódio ofertado na refeição é decorrente desses produtos, como apresentado no Quadro I.

Quadro I. Contribuição dos alimentos ultraprocessados no total calórico e quantidade de sódio no lanche da tarde de duas semanas em uma UAN escolar, São Paulo, 2016.

\begin{tabular}{|c|c|c|c|c|c|c|}
\cline { 2 - 7 } \multicolumn{1}{c|}{} & \multicolumn{2}{c|}{ Quantidade total de kcal } & \multicolumn{2}{c|}{ Quantidade total de sódio } \\
\cline { 2 - 7 } & $\begin{array}{c}\text { Kcal do } \\
\text { cardápio }\end{array}$ & $\begin{array}{c}\text { Kcal } \\
\text { ultraproces- } \\
\text { sados }\end{array}$ & $\begin{array}{c}\text { Ultraproces- } \\
\text { sados \% }\end{array}$ & $\begin{array}{c}\text { Na do } \\
\text { cardápio } \\
\text { (mg) }\end{array}$ & $\begin{array}{c}\text { Ultraproces- } \\
\text { sados }\end{array}$ & $\begin{array}{c}\text { \% nos } \\
\text { ultraproces-sados }\end{array}$ \\
\hline Semana 1 & $1.729,6$ & 983,3 & 56,9 & 1.355 & 972,6 & 71,8 \\
\hline Semana 2 & $1.764,8$ & 1002,6 & 56,8 & 1.774 & 1.347 & 75,9 \\
\hline
\end{tabular}

Fonte: do autor.

O valor calórico do lanche variou de $182,2 \mathrm{kcal}$ a 504,6kcal,sendo a média da densidade calórica do cardápio das duas semanas de $349,4 \mathrm{kcal}$, das quais $198,6 \mathrm{kcal}$ (desvio padrão = $\pm 104)$ sãode alimentos ultraprocessados.

Segundo o Programa Nacional de Alimentação Escolar (PNAE) o conteúdo dos lanches oferecidos aos escolares não deverá ser superior a $20 \%$ da recomendação nutricional diária (SBP, 2012). Embora a escola não esteja cadastrada no programa, neste estudo utilizou-se a recomendação de 15 a $20 \%$ das necessidades diárias para análise da densidade calórica, como preconiza o PNAE.

Nota-se que apenas um dia da semana 1 encontra-se no valor adequado, enquanto os outros variam para mais ou para menos da recomendação. Já na semana 2, três dias encontram-se dentro do recomendado, como visto no quadro II. 
Quadro II - Distribuição energética dos lanches da tarde oferecidos em uma UAN escolar, com base do valor energético diário de $2.000 \mathrm{kcal}$ ou $8.400 \mathrm{Kj}$.

\begin{tabular}{|l|c|c|c|c|c|}
\cline { 2 - 5 } \multicolumn{1}{c|}{} & Segunda & Terça & Quarta & Quinta & Sexta \\
\hline Semana 1 & $20,8 \%$ & $10,8 \%$ & $16,7 \%$ & $23,7 \%$ & $14,4 \%$ \\
\hline Semana 2 & $16,9 \%$ & $20,0 \%$ & $9,11 \%$ & $15,9 \%$ & $25,2 \%$ \\
\hline
\end{tabular}

Fonte: do autor.

\section{DISCUSSÃO}

Foi possível verificar que há uma contribuição muito significativa de alimentos ultraprocessados, tanto na densidade energética quanto de sódio nos cardápios avaliados. A porcentagem de quilocalorias provenientes de ultraprocessados nas duas semanas foi de aproximadamente $57 \%$, enquanto que a média de porcentagem de sódio proveniente de ultraprocessados foi de aproximadamente $74 \%$.

Entretanto, é importante frisar que as refeições são de livre demanda, ou seja, teoricamente a criança pode pegar a quantidade que ela quiser de produtos, alterando para mais ou para menos a quantidade de sódio e calorias neste caso.

A última versão do Guia Alimentar para a População Brasileira, lançada no ano de 2014 recomenda que a dieta deve ser composta basicamente pelos alimentos in natura ou minimante processados, e que o consumo de alimentos ultraprocessados seja restrito, devido à alta quantidade de calorias, sódio, açúcares e gordura, que podem contribuir para o excesso de peso e obesidade, bem como para o desenvolvimento de doenças crônicas na vida adulta, além de que uma má alimentação pode ser prejudicial no rendimento escolar (BOCCALETTO e MENDES, 2009; LOUZADA,
2015). No presente estudo, foi possível verificar uma participação importante dos alimentos ultraprocessados no cardápio.

Com relação ao sódio, a Organização Mundial da Saúde (OMS) recomenda uma ingestão máxima diária de 2000mg (2g) por dia, tanto para adultos como para crianças, o que corresponde a $5 \mathrm{~g}$ de cloreto de sódio (sal de cozinha) por dia. Segundo dados da Pesquisa de Orçamentos Familiares - POF 2008/2009, estimase que a população brasileira, em geral consome $o$ dobro do recomendado pela OMS (BRITO e SPINELLI, 2016).

$\mathrm{O}$ aumento do consumo de alimentos ultraprocessados pode ser um dos fatores que interferem na ingestão excessiva de sódio por dia (BOCCALETTO E MENDES, 2009). Os achados do presente estudo constatam que a contribuição de sódio sobre o total oferecido na refeição é de 74\% aproximadamente, ou seja, grande parte do sódio consumido no lanche é proveniente de alimentos ultraprocessados. Lembrando que, neste estudo foi analisado apenas o cardápio do lanche da tarde, ou seja, não levou-se em consideração o restante das refeições ofertadas na instituição, e sem contar o que os escolares consomem fora do ambiente escolar.

Este dado é preocupante, e reflete um perfil alimentar encontrado cada dia mais em evidência na população brasileira, segundo 
diversos estudos (BIELEMANN, 2015; MARTINS et al., 2013). Além disso, as crianças se interessam mais por alimentos ultraprocessados, devido à maior densidade energética e palatabilidade (BOCCALETTO e MENDES, 2009).

É de suma importância a análise da composição de cardápios oferecidos nas escolas. Entretanto, são necessários mais estudos que avaliem estes parâmetros. Um fator limitante do trabalho foi a ausência de outros trabalhos para comparar os dados encontrados.

\section{CONCLUSÃO}

Pode-se concluir que é grande a quantidade de alimentos ultraprocessados oferecida na instituição avaliada. É importante ressaltar que este grupo de alimentos, quando consumidos em excesso podem ser prejudiciais à saúde do indivíduo, e contribuem para o aumento da densidade calórica e do teor de sódio na refeição.

Cabe aos serviços de alimentação a adequação dos cardápios, de forma que haja a oferta de alimentos e preparações equilibradas nutricionalmente, com o objetivo de proteger à saúde dos comensais, além de ser uma forma de estimular hábitos alimentares mais saudáveis, principalmente na infância, pois sabe-se que a escola possui grande influência na formação dos hábitos alimentares das crianças.

\section{REFERÊNCIAS BIBLIOGRÁFICAS}

ABREU, E.S.; SPINELLI, M.G.N.; SOUZA PINTO, A.M. Gestão de Unidades de Alimentação e Nutrição: um modo de fazer. 6. ed. São Paulo: Metha, 2016,392p.

ALVES, F.A. A organização da produção de Unidades de Alimentação e Nutrição. Dissertação para obtenção de título de Mestre em Administração, Universidade Federal de Santa Catarina, Florianópolis, 2005.

BIELEMANN, R.M. et al.; Consumo de alimentos ultraprocessados e impacto na dieta de adultos jovens. Rev Saúde Pública 2015;49:28.

BOCCALETTO, E.M.A; MENDES, R.T. (organizadores). Alimentação, atividade física e qual idade de vida dos escolares do município de Vinhedo/SP. Campinas: IPES Editorial, 2009. Disponível em: <http://www.bibliotecadigital. unicamp.br/document/?down=000468112>.

BRASIL. IBGE - Instituto Brasileiro de Geografia e Estatística. Pesquisa de Orçamentos Familiares 2008-2009 - POF. 2009.

BRASIL. RESOLUÇÃO CNS N ${ }^{\circ} 510$, de 07 de abril de 2016. Ética na Pesquisa na área de Ciências Humanas e Sociais. Diário Oficial da União.Brasília, DF, 24 de maio de 2016. Disponível em: <https://www. conselho.saude.gov.br/resolucoes/2016/Reso510.pdf $>$.

BRITO, A.M.O; SPINELLI, M.G.N. Oferta de sódio oriundo de alimentos industrializados em restaurantes institucionais. Demetra; 2016; 11(2); 321-336. Disponível em: <http://www.epublicacoes.uerj.br/index.php/demetra/article/view/ 16779>.

BUENO, A.L.C. et al. Blog UAN Escolar. Apresentação - O que é uma UAN? Novembro, 2015. Disponível em: <https://uanescolar.wordpress.com/2015/11/24/ apresentacao-o-que-e-uma-uan/>.

FLÁVIO, E.F. Alimentação escolar e avaliação nutricional dos alunos do ensino fundamental das escolas municipais de Lavras, MG. Tese para obtenção de título para "Doutor". Lavras, Minas Gerais, 2006.

LOUZADA, M.L.C et al. Alimentos ultraprocessados e perfil nutricional da dieta no Brasil. Rev. Saúde Pública, 2015;49:38

MARTINS, A.P.B et al . Participação crescente de produtos ultraprocessados na dieta brasileira (1987- 
2009). Rev. Saúde Pública, São Paulo, v. 47, n. 4, p. 656-665, Ago. 2013.

MONTEIRO, C.A. et al.; Guia Alimentar para a população brasileira. Ministério da Saúde, Secretaria de Atenção à Saúde, Departamento de Atenção Básica - 2. ed. Brasília: Ministério da Saúde, 2014.

PAIVA, X.V. Qualidade nutricional dos lanches escolares das escolas particulares do Plano Piloto DF 009. 67 f. Dissertação (Mestrado em Nutrição Humana)_-Universidade de Brasília, Brasília, 2009.

PHILIPPI, S T. Tabela de composição de alimentos: suporte para decisão nutricional. $2^{\mathrm{a}}$ Edição.

Anvisa/finatec/nut Unb.

Sociedade Brasileira de Pediatria (SBP) - Lanche Saudável - Manual de orientação. Departamento Científico de Nutrologia, 2012. Disponível em:

<http://www.sbp.com.br/pdfs/Manual_Lanche_saudave 1_04_08_2012.pdf>

Tabela Brasileira de Composição de Alimentos TACO. 4. ed. Campinas, SP: NEPA-UNICAMP, 2011.

VIEIROS, M.B.; MARTINELLI, S.S. Avaliação Qualitativa das Preparações do Cardápio Escolar AQPC Escola. Rev. Nutrição em Pauta, Ano 20 número 114 - maio/junho/2012.

WORLD HEALTH ORGANIZATION. Guideline: Sodium intake for adults and children. Geneva, World Health Organization (WHO), 2012. 18-19. Disponível em:

<http://apps.who.int/iris/bitstream/10665/77985/1/9789 241504836_eng.pdf?ua=1\&ua=1>

\section{Yasmin Ferreira Alves}

Graduanda de Nutrição pela Universidade Paulista UNIP campus Cidade Universitária.

Caroline Regina da Silva

Graduanda de Nutrição pela Universidade Paulista UNIP campus Cidade Universitária.

Mônica Glória Neumann Spinelli

Doutora em Saúde Pública pela Universidade de São Paulo, docente na Universidade Paulista - UNIP. 